\title{
Scoring Systems in Acute Appendicitis - A Review
}

\author{
Aditya Vijay Mundada ${ }^{1}$, Yeshwant Lamture ${ }^{2}$, Meenakshi Yeola ${ }^{3}$ \\ 1, 2, 3 Department of General Surgery, Jawaharlal Nehru Medical College, \\ Datta Meghe University of Medical Sciences, Wardha, Maharashtra, India.
}

\section{ABSTRACT}

Acute appendicitis is the most frequent cause leading to emergency laparotomy. In developing countries with resource limited settings, diagnosing appendicitis in its acute from is a challenge as clinical examination remains the cornerstone of diagnosis due to lack advanced radiological investigations and cost restraints. Presently, various modalities are in use to aid in indeterminate cases to decrease negative appendectomy rates. Scoring systems are amongst them. These systems take advantage of clinical history, physical signs and symptoms and laboratory findings. This article will recognize the widely used scoring systems and their accuracy and limitations in diagnosing acute appendicitis in adults.

32 studies have been included in this review till June 2020. A collective review of these studies regarding the application of scoring systems and their statistical significance in diagnosing acute appendicitis along with their limitations has been discussed and its conclusion noted in this review article.

All the widely used scoring systems have some limitations in diagnosing acute appendicitis. Thus, the only way to decrease the morbidity and mortality associated with appendicitis is to make an ideal scoring system which is simple, reliable and cost effective.

\section{KEY WORDS}

Appendicitis, Scoring System, Appendicectomy, Clinical Predictive Rules, Right Iliac Fossa Pain, Right Lower Quadrant Pain, Guidelines
Corresponding Author: Dr. Yeshwant Lamture, Professor, Staff Quarters, JNMC Campus, Sawangi, Wardha, Maharashtra-442 001, India.

E-mail: dryrlamture@yahoo.co.in

DOI: $10.14260 /$ jemds/2020/850

How to Cite This Article:

Mundada AV, Lamture Y, Yeola M. Scoring systems in acute appendicitis - a literature review. J Evolution Med Dent Sci 2020;9(51):3881-3886, DOI: $10.14260 /$ jemds/2020/850

Submission 24-08-2020,

Peer Review 27-10-2020,

Acceptance 04-11-2020,

Published 21-12-2020.

Copyright (C) 2020 Aditya Vijay Mundada et al. This is an open access article distributed under Creative Commons Attribution License [Attribution 4.0 International (CC BY 4.0)] 


\section{BACKGROUND}

Acute appendicitis is the most frequent cause leading to emergency laparotomy. In developing countries, with resource limited settings, diagnosing appendicitis in its acute from is a challenge as clinical examination remains the cornerstone of diagnosis due to lack of advanced radiological investigations and cost restraints. Diagnosis becomes more difficult in the elderly and in female patients who are in the reproductive age group due to the presence of atypical symptoms leading to negative appendectomy. A negative appendectomy is the surgery done for a clinically diagnosed appendicitis resulting in a normal appendix on histopathology. Various modalities have been used in uncertain cases in efforts to minimize negative appendectomies. Scoring systems are amongst them. These systems take advantage of clinical history, physical signs and symptoms and laboratory findings.

Despite of advances, there has not been any breakthrough in diagnosing appendicitis in a reliable, cost effective and accurate way. It is least in extremes of age. Scoring systems are of immeasurable value in distinguishing vague abdominal pain from acute appendicitis. Several scores have been in use to aid physicians in diagnosing appendicitis without raising the number of perforations and to reduce the number of harmful appendectomies. ${ }^{1}$

The aim of this study was to recognize the widely used clinical predictive rules and their accuracy in diagnosing acute appendicitis in adults.

\section{METHODS}

\section{Search Strategy}

A comprehensive set of keywords or search terms were built like appendicitis, scoring system, appendicectomy, clinical predictive rules, right iliac fossa pain, right lower quadrant pain, guidelines. We searched Medline, Scopus, Google scholar, Web of Science, PubMed, Cochrane Central Register of Controlled Trials databases and selected relevant observational studies up to June 2020. The list of reference articles was retrieved and they were searched via automation and manually. Studies were restricted to English language and humans only.

\section{Data Extraction}

Data extraction was done by two authors into separate sheets on Microsoft excel 2020. The differences between the two authors on selection of articles and data extraction were sorted through discussion. There was no restriction set on the date of publication of the articles. Automated \& manual reduplications was performed.

\section{Inclusion Criteria}

Those research studies were selected which derived a scoring system or assessed the efficacy of a scoring system for use in the adult population with right iliac fossa pain. A scoring system was defined as the one which 2, 3

- Included three or more factors derived from the history, clinical examination or laboratory tests.
- Helped in decision making or guided in planning the treatment.

- Was not a practice guideline.

A study deriving a scoring system was the one which explained the formation and its application in clinical setting. Similarly a study validating a scoring system assessed its performance with reference to its sensitivity, specificity, area under the curve and negative appendicectomy rate in the general population

\section{Exclusion Criteria}

The scores which were used only for a specific group of population like paediatric, geriatric were excluded. The studies which did not assess the performance of a scoring system were excluded. Studies including pregnant patients and patients with chronic appendicitis were excluded.

\section{VARIOUS SCORING SYSTEMS}

The Alvarado score, the Modified Alvarado score, the RIPASA score, Tzanakis score are amongst the well-known scores. The other scores with low outcome and less known are the Appendicitis Inflammatory Response score, Christian score and Izbicki score. These tools can be helped to diagnose and differentiate the patients who need surgical intervention from the ones who can be managed conservatively with intravenous antibiotics, supportive care and monitoring.

\section{Alvarado Score}

Alvarado in 1986 created a clinical scoring system comprising of various symptoms, signs and laboratory parameters frequently seen in patients with acute appendicitis. The score is interpreted as follows -

- 5 - 6 score: Conservative management.

- 7 - 8 score: Observe the patient and repeat scoring.

- 9 - 10 score: Surgery. ${ }^{2}$

\begin{tabular}{|c|c|}
\hline Signs & 2 \\
Right iliac fossa tenderness & 1 \\
Raised temperature $\left(>99.1^{\circ} \mathrm{F}\right)$ & 1 \\
Blumberg sign (rebound tenderness) & 1 \\
Symptoms & 1 \\
Anorexia & 1 \\
Nausea or vomiting & 2 \\
Migration of pain to right lower quadrant & $\mathbf{1}$ \\
Laboratory Values & \\
Leukocytosis ( $>10,000 \mathrm{WBC})$ & \\
\hline Lift shift ( $>75 \%$ neutrophils) & Figure 2 . The Alvarado Score \\
\hline
\end{tabular}

It has been agreed that the Alvarado score is safe and uncomplicated. It helps the surgeon to make a correct diagnosis of appendicitis thereby giving optimum treatment.

\section{Izbicki Score ${ }^{3}$}

Izbicki in 1990 proposed a score which included 7 parameters namely sex, white blood cell counts, guarding, rebound tenderness and characteristics of pain such as its migration, 
duration and type. A score of more than two indicates that by patient should undergo surgery.

\begin{tabular}{|cclcl|}
\hline Gender & Male & $\mathbf{1}$ & Female & $\mathbf{0}$ \\
White cell count & $\begin{array}{c}\text { 11,000 x } 10^{\wedge} 9 \\
\text { / L }\end{array}$ & 1 & $<11,000$ & 0 \\
Guarding & Present & 1 & Absent & 0 \\
$\begin{array}{c}\text { Rebound Pain } \\
\text { Migration of } \\
\text { pain to right } \\
\text { lower quadrant }\end{array}$ & Present & 1 & Absent & 0 \\
$\begin{array}{c}\text { Pain duration } \\
\text { Character of } \\
\text { Pain }\end{array}$ & Present & 1 & Absent & 0 \\
\hline Intermittent & 1 & Figure 3. The Izbicki Score & 0 \\
\hline
\end{tabular}

\section{Modified Alvarado Scoring System (MASS) ${ }^{4}$}

Kalan et al in 1994 modified the already existing Alvarado score by removing tone parameter of 'shift to left of neutrophils'.

\begin{tabular}{|cc|}
\hline Items & Score \\
\hline Migratory RIF pain & 1 \\
Anorexia & 1 \\
Nausea or Vomiting & 1 \\
Tenderness in iliac fossa on right side & 2 \\
Rebound tenderness in the right lower & 1 \\
quadrant & 1 \\
Raised temperature $\left(>37.3^{\circ} \mathrm{C}\right)$ & 2 \\
\hline Raised WBC $(>10000 / \mathrm{ml})$ & Figure 4. Modified Alvarado Score \\
\hline
\end{tabular}

\section{Ohmann Score ${ }^{5}$}

This score was developed in Germany in 1995. This scoring system included the following signs and symptoms along with its respective value.

\begin{tabular}{|cc|}
\hline Sign / Symptom & Score \\
Tenderness in the right lower quadrant & 4.5 \\
Rebound tenderness & 2.5 \\
Urinary symptoms - Absent & 2.0 \\
Continuous pain & 2.0 \\
WBC count $\geq 10000 / \mu \mathrm{IL}$ & 1.5 \\
Age below 50 years & 1.5 \\
Migratory RIF pain & 1.0 \\
Involuntary muscular tension & 1.0 \\
\hline \multicolumn{2}{|c}{ Figure 5. Ohmann Score } \\
\hline
\end{tabular}

\section{Tzanakis Scoring System}

Tzanakis et al. ${ }^{6}$ in 2005 made a new scoring system for appendicitis to help in diagnosing appendicitis. It includes four variables made up of various signs and symptoms along with ultrasonography. A score of eight or more out of 15 points is suggestive of appendicitis. The score is as follows:

\begin{tabular}{|cc|}
\hline Parameters & Score \\
\hline Tenderness in right iliac fossa & 4 \\
Rebound tenderness & 3 \\
Leucocytosis & 2 \\
Ultrasonography & 6 \\
Total & 15 \\
\hline \multicolumn{2}{c}{ Figure 6. Tzanakis Score } \\
\hline
\end{tabular}

\section{Lintula Scoring System ${ }^{7}$}

The Lintula score was first developed in 2005 for use in children but later it has been validated in adults too in diagnosing acute appendicitis. Interpretation of score is as follows -

- $\quad$ Score 15 and below - No appendicitis

- Score 21 and above - Appendicitis present.

\section{Appendicitis Inflammatory Response Score ${ }^{8}$}

The Appendicitis Inflammatory Response (AIR) score separates the patients into 3 groups namely low, medium, or high probability of acute appendicitis. It is based on the same principles as that of Alvarado score. It was created by Andersson and Andersson in 2008. The score was formed by incorporating 8 variables. The drawback of the score is that it has excluded a very important sign of migratory right iliac fossa pain

\section{The RIPASA Scoring System ${ }^{9}$}

The Raja Isteri Pengiran Anak Saleha Appendicitis score made in 2010 is a clinical predictive rule which has been made for South Asian population and includes 18 fixed variables (four demographics, six symptoms, five signs and two investigations and NRI status)

\section{DISCUSSION}

Khadda and colleagues ${ }^{10}$ recorded that the RIPASA score was $97.7 \%$ sensitive and $77.4 \%$ specific in diagnosing acute appendicitis. He registered a NAR of $13.7 \%$ which was higher than the NAR of $1.9 \%$ reported by Menon et al ${ }^{11}$ in Pakistan. In another analysis, using the RIPASA score, Pouget-Baudry et al ${ }^{12}$ recorded a NAR of $1.72 \%$ in France. Khadda considers the Alvarado score to be the most user friendly of all the scores currently used.

In addition, Gaikwad et $\mathrm{l}^{13}$ recorded a specificity of $100 \%$ on the addition of ultrasonography to the Alvarado score in India. In India, Goel et al ${ }^{14}$ did a comparative study which included Alvarado and RIPASA scores and found that the Alvarado score was superior to the RIPASA score in terms of specificity (100 versus $50 \%$ ). The NAR of RIPASA was higher than the NAR of Alvarado score ( $5 \%$ versus $0 \%$ ). The findings were comparable to the study performed in Iran by Karami et al ${ }^{15}$ who considered the Alvarado score to be $100 \%$ specific when compared to the RIPASA and AIR score (91.6\% specificity for both).

In Ireland, Malik et al ${ }^{16}$ reported getting a PPV and NPV of $84.06 \%$ and $72.86 \%$ respectively for the RIPASA score. He recorded a NAR of $15.94 \%$ and $80 \%$ diagnostic accuracy. This was the first study which assessed the usefulness of the RIPASA score in the diagnosis of acute appendicitis in the western population. In India, however, Rodrigues and Sindhu ${ }^{17}$ stated that the Alvarado score was more precise than the RIPASA score, and had better PPV. Compared to other studies in which NAR ranged from zero to $10 \%$ for Alvarado score, the NAR in this study was more (18.09\%). Rathod et al ${ }^{18}$ reported similar findings in his RIPASA score (Raja Isteri Pengiran Anak Saleha Appendicitis) analysis with a NAR (negative appendectomy rate) of $20.69 \%$ and a rate of perforated appendicitis of $8.05 \%$. This suggests that, at the 
cost of a high NAR, the RIPASA score will reduce the occurrence of perforated and gangrenous appendicitis.

In a recent analysis in India, Regar et al ${ }^{19}$ observed that the specificity of Alvarado score (80\%) was greater than that of RIPASA score (60\%). Similarly, the PPV (positive predictive value) of Alvarado score (98.46\%) was slightly better than the PPV of RIPASA score (97.83\%). The NAR of Alvarado score was lower than that of the RIPASA score (1.54 vs. $2.17 \%$ ).

In India, Sinnet et al 20 noticed that the RIPASA score was more sensitive ( $95 \%$ vs. $65 \%$ ) but less specific ( $65 \%$ vs. 90 $\%)$ than the Alvarado score in evaluating acute appendicitis. The PPV was $92.89 \%$ for the RIPASA score and $96.6 \%$ for the Alvarado score indicating that the NAR of Alvarado score was lower than that of the RIPASA (3.33\% vs. $7.61 \%$ ).

Erdem et $\mathrm{al}^{21}$, in his study done in Turkey assessed the reliability and practicality of RIPASA, Eskelinen, Ohmann and Alvarado score and reported that the Alvarado score had the least NAR of $12 \%$ amongst other scores included in the study. The RIPASA score had a NAR of $25 \%$. The NAR for the Ohmann score was $22 \%$ and for the Eskelinen score was $21 \%$.

Diaz-Barrientos et al 22 in his study in Mexico reported that there was no significant difference between the RIPASA and the modified Alvarado score when ROC curve (receiver operating characteristic curve) area was considered as it was 0.59 for the RIPASA score and 0.71 for the Alvarado score. Another study was done by Reyes-Garcia et $\mathrm{al}^{23}$ and he reported that the NAR for RIPASA score was very high (18.6 $\%)$ and there were more percentage of complicated appendicitis in the form of necrotizing appendix in $15.7 \%$ cases and perforated appendix in $15.7 \%$ of cases on application of RIPASA score.

The physician determined decision was compared with RIPASA, Alvarado and MASS scoring systems (Modified Alvarado Scoring System) by Golden et $\mathrm{al}^{24}$ so as to estimate the physician gestalt in diagnosing appendicitis. They reported that RIPASA had high sensitivity (78 \%) and Alvarado had low sensitivity (47\%) at a higher cut off value. Diametrically, Alvarado had high specificity (81\%) than the RIPASA (36\%) at a higher cut off value. The test attributes of Alvarado score was between these two values. Test characteristics were also measured at the lower rule out value for the scoring systems which showed that negative predictive value of MASS (75\%) was less than negative predictive value of RIPASA score $(89$ $\%$ ). Physician gestalt had a negative predictive value of $83 \%$. RIPASA score had the least area under the curve (AUC) of 67 $\%$. The AUC (area under the curve) was highest for the physician-determined decision as well as for the Alvarado score $(72 \%)$ and intermediate for MASS (70 \%). These researchers established that the accuracy of physician gestalt is comparable to the accuracy of these scoring systems and that physician gestalt is competent enough in diagnosing acute appendicitis.

Sammalkorpi et al ${ }^{25}$ made a scoring system by logistic regression called the Adult Appendicitis score with the help of multiple attributions for absent values. The efficacy of the score in terms of sensitivity and specificity was same as that of the Alvarado score. The Adult Appendicitis score had a better value of area under the ROC curve $(0.882)$ than that of the Alvarado score (0.790). The NAR of this new score was $18.2 \%$ which was way higher than conventional reported NAR for the Alvarado score.
A prospective study on the comparison of Tzanakis score with the Alvarado score was done by Sigdel GS et al $^{26}$ where he reported that the result of Tzanakis score was better than Alvarado score with reference to its sensitivity $(91.4 \%$ for Tzanakis versus $81 \%$ for Alvarado). Both the scores had same specificity of $66.6 \%$. The AUC of Tzanakis (0.867) was slightly higher than that of the Alvarado (0.81). Sigdel reported a NAR of $6 \%$ for Tzanakis scoring system which is quite lower than the accepted range of $15-25 \%$. This low rate of negative appendectomies was attributed to the addition of ultrasonography in the scoring system which in contrast is not included in other scoring systems due to its non-availability in the resource limited settings. Tzanakis score had a better diagnostic accuracy of $91.48 \%$ than the diagnostic accuracy of Alvarado score which was $81.91 \%$.

A study was conducted in India by Sharma et al where he compared the outcome of Tzanakis score with the outcome of Modified Alvarado score with regards to its sensitivity, specificity and diagnostic accuracy. He found that Tzanakis had a low sensitivity of $82 \%$ as compared to the sensitivity of MASS which was $97.7 \%$. Conversely, Tzanakis score had specificity of $36.38 \%$ and MASS had specificity of $18 \%$. This showed that Tzanakis was more specific than MASS. Both the scoring system had same PPV of $19 \%$. MASS was more accurate than Tzanakis scoring system in diagnosing acute appendicitis (89\% vs. $79 \%$ ). The study concluded that MASS had a better outcome than the Tzanakis scoring system. This difference in outcome was due to inter-observer bias observed during the ultrasonography. Another disadvantage of Tzanakis score was the inclusion of criteria of raised leukocyte count defined as WBC counts more than 12,000 cells $/ \mathrm{cm}^{3}$ as most number of patients who present with uncomplicated appendicitis had normal or mildly raised WBC counts.

Similar results were derived by Kumar et al in his study on Tzanakis scoring system ${ }^{27}$ in India. He found that the Tzanakis score is a valid, accurate and efficacious modality in diagnosing acute appendicitis but the only limitation was inter-observer bias encountered during ultrasonography.

A study comparing Alvarado score with Lintula score in patients with age more than 75 years was done by Konan et al 28 and found that Alvarado score had better precision in diagnosing appendicitis than the Lintula score. He also reported that both the scores were highly sensitive and specific in diagnosing appendicitis.

Similarly another prospective study done by Ojuka and Sangoro $^{29}$ reported that the ROC curve for Lintula score is quite similar to that of Alvarado scores (0.6824 and 0.6966). However, Lintula score was less sensitive than the Alvarado score (60.8 vs. $83.3 \%$ ). They also reported that Alvarado score had a better diagnostic accuracy than that of Lintula score (70.4\% vs. $69.6 \%$ ).

Horzic et $\mathrm{al}^{30}$ analysed and compared the modified Alvarado, Ohmann and Eskelin scores in diagnosing acute appendicitis and found that every patient with the modified Alvarado score of greater than seven had features of inflamed appendix on histopathology. This showed that modified Alvarado score had a specificity of $100 \%$ in this study. Thus, the authors concluded that MASS can be used as a tool to determine the need for urgent laparotomy. 


\section{CONCLUSIONS}

An ideal clinical prediction rule for acute appendicitis should be basic, fast, convenient to use, cost efficient and reliable. It should contain elements with a good statistical significance. It should be able to reduce the rate of negative appendicectomy and decrease the inadvertent wastage of useful resources and manpower in a resource limited setting. The need for developing a good scoring system to be applicable in all age groups is the need of the hour in this $21^{\text {st }}$ century.

Financial or other competing interests: None.

Disclosure forms provided by the authors are available with the full text of this article at jemds.com.

\section{REFERENCES}

[1] Kularatna M, Lauti M, Haran C, et al. Clinical prediction rules for appendicitis in adults: Which is best? World J Surg 2017;41(17):1769-81.

[2] Alvarado A. A practical score for the early diagnostic of acute appendicitis. Annals of Emergency Medicine 1986;15(5):557-64.

[3] Izbicki JR, Knoefel WT, Wilker DK, et al. Accurate diagnosis of acute appendicitis: a retrospective and prospective analysis of 686 patients. Eur J Surg 1992;158(4):227-31.

[4] Kalan M, Talbot D, Cunliffe WJ, et al. Evaluation of the modified Alvarado score in the diagnosis of acute appendicitis: a prospective study. Annals of the Royal College of Surgeons of England 1994;76(6):418-9.

[5] Ohmann C, Franke C, Yang Q. Clinical benefit of a diagnostic score for appendicitis: results of a prospective interventional study. Archives of Surgery 1999;134(9):993-6.

[6] Tzanakis NE, Stamatis P, Danulidis K, et al. New approach to accurate diagnosis of acute appendicitis. World Journal of Surgery 2005;29(9):1151-7.

[7] Lintula H, Kokki H, Ketunnen R, et al. Appendicitis score for children with suspected appendicitis. A randomized clinical trial. Langenbeck's Archives of Surgery 2009;294(6):999-1004.

[8] Andersson M, Andersson RE. The appendicitis inflammatory response score: a tool for the diagnosis of acute appendicitis that outperforms the Alvarado score. World Journal of Surgery 2008;32(8):1843-9.

[9] Chong CF, Adi MIW, Thien A, et al. Development of the RIPASA score: a new appendicitis scoring system for the diagnosis of acute appendicitis. Singapore Medical Journal 2010;51(3):220-5.

[10] Khadda S, Yadav AK, Anwar A, et al. Clinical study to evaluate the RIPASA scoring system in the diagnosis of acute appendicitis. American Journal of Advanced Medical and Surgical Research 2015;1(2):67-73.

[11] Menon AA, Vohra LM, Khalik T, et al. Diagnostic accuracy of Alvarado score in the diagnosis of acute appendicitis. Pakistan Journal of Medical Sciences 2009;25(1):118-21.

[12] Pouget-Baudry Y, Mucci S, Eyssartier E. Le score clinicobiologique d'Alvaradodans la prise en charge d'unedouleur de fosse iliaquedroite chez l'adulte. Journal De Chirurgie Viscerale 2010;147(2):128-32.
[13] Gaikwad V, Murchite S, Chandra S, et al. To evaluate the efficacy of Alvarado score and ultrasonography in acute appendicitis. IOSR Journal of Dental and Medical Sciences 2016;15(9):14-8.

[14] Goel P, Mishra KR, Sharma R, et al. The study to evaluate the efficacy of Alvarado score and RIPASA score in diagnosis of acute appendicitis and correlation with intraoperative and pathological findings. Annals of Medical and Dental Research 2017;3(6):33-9.

[15] Karami MY, Niakan H, Zabedagheri N, et al. Which one is better? Comparison of acute inflammatory response, Raja Isteri Pengiran Anak Saleha appendicitis and Alvarado scoring systems. Annals of Coloproctology 2017;33(6):227-31.

[16] Malik MU, Connelly TM, Awan F, et al. The RIPASA score is sensitive and specific for the diagnosis of acute appendicitis in a western population. International Journal of Colorectal Disease 2017;32(4):491-4.

[17] Rodrigues W, Sindhu S. Diagnostic importance of Alvarado score in acute appendicitis. International Journal of Scientific Study 2017;4(11):57-60.

[18] Rathod S, Ali I, Bawa APS, et al. Evaluation of Raja Isteri Pengiran Anak Saleha appendicitis score: a new appendicitis scoring system. Medical Journal of D.Y. Patil University 2015;8(6):744-9.

[19] Regar MK, Choudhary GS, Nogia C, et al. Comparison of Alvarado and RIPASA scoring systems in diagnosis of acute appendicitis and correlation with intraoperative and histopathological findings. International Journal of Surgery 2017;4(5):1755-61.

[20] Sinnet PR, Chellappa PM, Kumar S, et al. Comparative study on the diagnostic accuracy of the RIPASA score over Alvarado score in the diagnosis of acute appendicitis. Journal of Evidence Based Medicine Healthcare 2016;3(80):4318-21.

[21] Erdem H, Cetinkünar S, Das K, et al. Alvarado, Esleinem, Ohmann and Raja Isteri Pengiran Anak Saleha appendicitis scores for diasgnosis of acute appendicitis. World Journal of Gastroenterology 2013;19(47):9057-62.

[22] Diaz-Barrientos CZ, Aquino-Gonzalez A, HerediaMontaño M, et al. Escala RIPASA para el diagnóstico de apendicitisaguda: comparación con la escala de Alvarado. Revista de Gastroenterología de México 2018;83(2):1126.

[23] Reyes-Garcia N, Zaldivar-Ramirez FR, Cruz-Martinez R, et al. Precisión diagnóstica de la escala de RIPASA para el diagnóstico de appendicitis aguda: análisis comparativo con la escala de Alvarado modificada. Cirujano General 2012;34(2):101-6.

[24] Golden SK, Harringa JB, Pickhard PJ, et al. Prospective evaluation of the ability of clinical scoring systems and physician-determined likelihood of appendicitis to obviate the need for CT. Emergency Medicine Journal 2016;33(7):458-64.

[25] Sammalkorpi HE, Mentula P, Leppaniemi A. A new adult appendicitis score improves diagnostic accuracy of acute appendicitis: a prospective study. BMC Gastroenterology 2014;14:114.

[26] Sigdel GS, Lakhey PJ, Mishra PR. Tzanakis score vs. Alvarado score in acute appendicitis. Journal of the Nepal Medical Association 2010;49(178):96-9. 
[27] Kumar SLA, Nagaraja AL, Srinivasaiah M. Evaluation of Tzanakis scoring system in acute appendicitis: International Journal of Surgery 2017;4(10):3338-43.

[28] Konan A, Hayran M, Kilic YA, et al. Scoring systems in the diagnosis of acute appendicitis in the elderly. Ulusal Travmave Acil Cerrahi Dergisi 2011;17(5):396-400.
[29] Ojuka D, Sangoro M. Alvarado vs. Lintula scoring system in acute appendicitis. The Annals of African Surgery 2017;14(1):22-8.

[30] Horzic M, Salamon A, Kopljar M, et al. Analysis of scores in diagnosis of acute appendicitis in women. Collegium Antropologicum 2005;29(1):133-8. 